\title{
On the Multiplicity of the Second Eigenvalue of Laplacian in $R^{2}$
}

\section{ZHANG LIQUN}

We prove the multiplicity of the second eigenvalue is at most two for a bounded smooth domain in $R^{2}$, which sharped an earlier result of Cheng, S.Y. and generalized a result of Lin, C.S.

\section{Introduction.}

We are concerned here with the eigenfunction of a domain $\Omega$ which is the solution of the following problems

$$
\left\{\begin{array}{l}
\Delta \phi+\lambda \phi=0 \text { in } \Omega \\
\left.\phi\right|_{\partial \Omega}=0
\end{array}\right.
$$

where $\Delta=\frac{\partial^{2}}{\partial x^{2}}+\frac{\partial^{2}}{\partial y^{2}}$ is the Laplacian. $\Omega$ is a bounded smooth domain in $R^{2}$, and $\lambda$ is the corresponding eigenvalue. Here $\Omega$ is smooth domain means that $\partial \Omega$ can be parameterized as a smooth function. Let $\lambda_{2}, \phi_{2}$ be the second eigenvalue and eigenfunction. It is well known that $\phi_{2}$ changes sign in $\Omega$. we know that the multiplicity of $\lambda_{2}$ is exactly two when the domain $\Omega$ is a disk and the corresponding eigenfunction can be written explicitly by Bessel functions. In [3], Cheng's arguments can be carried over to show that the multiplicity of the second eigenvalue of (1.1) is at most three. Later Lin in [5] sharpened his result by showing that the multiplicity is at most two provided $\Omega$ is convex. We will prove that the multiplicity of the second eigenvalue is at most two for any smooth domain.

Theorem 1.1. For a bounded smooth domain $\Omega$ in $R^{2}$, the multiplicity of second eigenvalues of the problem (1.1) is at most two.

This problem is closely related to the nodal line conjecture. Let $N\left(\phi_{2}\right)$ be the closure of $\left\{(x, y) \in \Omega \mid \phi_{2}(x, y)=0\right\}$ which is called the nodal line of $\phi_{2}$. It is easy to see that $N\left(\phi_{2}\right)$ is a regular curve at the point where $\left|\nabla \phi_{2}\right| \neq 0$. In fact, the singular set $S=\left\{(x, y) \in \Omega \mid \phi_{2}(x, y)=0\right.$ and $\left.\nabla \phi_{2}=0\right\}$ is 
composed of finite points in our case.(see Cheng [3]) Therefore $N\left(\phi_{2}\right)$ is regular except at finite points. The nodal line conjecture states that $N\left(\phi_{2}\right)$ can not be a closed curve. There are many works on this problem in the former literatures. Recently, Melas [8] proved the conjecture provided $\Omega$ is a convex domain. In the following, we will see that theorem 1.1 is a corollary of the nodal line conjecture in some case.

In this paper, we use an idea of "domain variation" which the author believe to be new in this field. When $\Omega$ is convex in one direction, theorem 1.1 can be obtained easily, because we can regard domain translations as a special variation in this case. In general, we consider a local variation of the domain and compare the two second eigenfunctions. Finally we prove the theorem by contradictions.

Remark 1.2. It remains to be an interesting open problem of the similar results in high dimensions.

Acknowledgement. The author thanks Prof. Ding W. Y. for many valuable discussions on this problem. He also thanks ICTP for the hospitability while he is visiting there and writing this paper.

\section{Preliminary Lemmas.}

In this section we present several basic lemmas. Although they have been proved before, we include their proof here for convenience of the reader. If $\psi$ is a solution of (1.1), by Taylor expansion at origin we know that

$$
\phi=C_{0}+C_{1} r \sin \left(\theta+\theta_{1}\right)+O\left(r^{2}\right) .
$$

where the first two leading terms are harmonic polynomials.

We call $\phi$ is first order vanishing at point $\mathrm{p}$, if $\phi(p)=0$ and second order vanishing at $\mathrm{p}$, if $\phi(p)=0, \nabla \phi(p)=0$. The following lemma is essentially contained in [3].

Lemma 2.1. The multiplicity of $\lambda_{2}$ of problem (1.1) in a bounded smooth domain is at most three.

Proof. Suppose that $\phi_{2}, \phi_{3}, \phi_{4}, \phi_{5}$ are orthogonal eigenfunctions corresponding to $\lambda_{2}$. Let $q \in \Omega$ be the origin of the coordinate. Choosing $c_{2}, c_{3}, c_{4}, c_{5}$, 
such that

$$
\phi=\sum_{i=2}^{5} c_{i} \phi_{i}
$$

satisfies

$$
\begin{gathered}
\phi(q)=0, \\
\nabla \phi(q)=0 .
\end{gathered}
$$

We can always choose $c_{i}, \mathrm{i}=2,3,4,5$, because there are only three equations. $\phi$ is an eigenfunction corresponding to $\lambda_{2}$ with a second order vanishing at point q. Then we have

$$
\phi(q)=c_{2} r^{2} \sin 2\left(\theta+\theta_{2}\right)+O\left(r^{3}\right),
$$

near the point $\mathrm{q}$. Then we can find a small circle centered at $\mathrm{q}$ on which $\phi$ changes sign at least four times. Thus at $\mathrm{q}, \phi$ has at least two nodal lines transversely intersect each other. Moreover, they divided $\Omega$ into at least three parts. From Courant nodal domain theorem, we know that the nodal line of $\phi$ divided $\Omega$ into exactly two parts. Therefore $\phi$ can not have a second order vanishing point in $\Omega$. Then we proved our lemma.

Remark 2.2. From the proof of lemma 2.1, we can see that the second eigenfunction can not have a second vanishing point in $\Omega$. Therefore the nodal line $N\left(\phi_{2}\right)$ is a regular curve in $\Omega$. But $N\left(\phi_{2}\right)$ may have a singular point on the boundary.

The following lemma gives us some nodal line behaviors on the boundary. It is essentially proved by Lin [5].

Lemma 2.3. Assume the multiplicity of $\lambda_{2}$ is three, then for any two points $p, q \in \partial \Omega$, there exists a second eigenfunction $\phi$ such that $N(\phi)$ intersects the boundary at these two points. Moreover, $p$ and $q$ are the second order vanishing points of $\phi$.

Proof. Suppose $\phi_{2}, \phi_{3}, \phi_{4}$ be three orthogonal second eigenfunctions. Again we let

$$
\phi=c_{2} \phi_{2}+c_{3} \phi_{3}+c_{4} \phi_{4}
$$


such that

$$
\frac{\partial \phi}{\partial n}(p)=0
$$

$$
\frac{\partial \phi}{\partial n}(q)=0
$$

where $\mathrm{n}$ is the normal direction. Such $\phi$ always exists, because there are two equations with three parameters. By Maximum principle we know that $N(\phi)$ intersects the boundary at $\mathrm{p}, \mathrm{q}$. Obviously $\mathrm{p}, \mathrm{q}$ are the second order vanishing points of $\phi$.

Under the assumptions of lemma 2.3, we have the following remarks.

Remark 2.4. If we choose $\mathrm{p}$ in lemma 2.3 be the origin of the coordinate and tangent direction be the $\mathrm{x}$-direction. We have the following expansion near $\mathrm{p}$

$$
\phi=c_{2} r^{2} \sin 2 \theta+O\left(r^{3}\right),
$$

where $c_{2} \neq 0$ if $\mathrm{p}, \mathrm{q}$ are different points and connected in $\partial \Omega$. And similar results hold for point $\mathrm{q}$. Moreover if $\mathrm{q}$ is in $\Omega$, we can also find an eigenfunction $\phi$, such that $N(\phi)$ passing through point p and q. In fact in this case (2.8) is replaced by $\phi(q)=0$.

Remark 2.5. If we let

$$
\frac{\partial^{2} \phi}{\partial n \partial T}(p)=0
$$

replace (2.8), where $\mathrm{T}$ is the tangent direction. Then $\mathrm{p}$ is a third order vanishing point of $\phi$. And $N(\phi)$ touches the boundary at p. In the coordinate as given in remark 2.4, we have the following local expansion

$$
\phi=c_{3} r^{3} \sin 3 \theta+O\left(r^{4}\right),
$$

where $c_{3} \neq 0$. In fact, if $c_{3}=0$ we can use a similar discussion as in the proof of lemma 2.1 to obtain a contradiction. That is the vanishing order is at most three.

Remark 2.6. If $\Omega$ is a simply connected domain, then there exists a second eigenfunction $\phi$, such that $N(\phi)$ is a closed curve. In fact, the function $\phi$ obtained in remark 2.5 is the desired eigenfunction. 
Corollary 2.7. If $\Omega$ is convex in one direction, then the multiplicity of $\lambda_{2}$ is at most two.

As we mentioned before, we have a shorter proof of theorem 1.1 in this case. And the idea of "domain variation" is developed from this proof.

Proof. Suppose the multiplicity of $\lambda_{2}$ is three. And we may assume that $\Omega$ is convex in x-direction. From remark 2.6, we know that there exists a second eigenfunction $\phi$ such that the nodal line of $\phi$ is closed.

Now, we consider $\phi_{x}$ which is the derivative of $\phi$ in $\mathrm{x}$-direction, it satisfies

$$
\Delta \phi_{x}+\lambda_{2} \phi_{x}=0
$$

Let $\mathrm{p}, \mathrm{q}$ be the maximum and minimum points in $\mathrm{y}$-direction on the boundary. Then $\phi_{x}$ is nonpositive on one part of $\widehat{p q}$ and nonnegative on the other of $\widehat{p q}$. And $\phi_{x}$ is not identically zero on the boundary.

Let $\psi$ be the second eigenfunction whose nodal line intersects the boundary at p,q. In the case of simply connected domain $N(\psi)$ intersects the boundary at exactly two points p,q. Then by compare (2.12) with (1.1), we have

$$
\int_{\partial \Omega} \phi_{x} \frac{\partial \psi}{\partial n}=0
$$

This is impossible, because $\phi_{x}$ and $\frac{\partial \psi}{\partial n}$ change sign simultaneously at points $\mathrm{p}, \mathrm{q}$.

Finally we should mention that theorem 1.1 is a corollary of nodal line conjecture provided $\Omega$ is simply connected domain. That is, if the nodal line conjecture is true, then our theorem is also true in a simply connected domain. This can be seen easily from remark 2.6 .

\section{Domain Variations.}

In this section, we sometime denote $\Omega$ by $\Omega_{0}$. Let $\Omega_{t}$ be a variation of $\Omega_{0}$ for some small $\mathrm{t}$. That is, $\partial \Omega_{t}$ as $\mathrm{t}$ varying, is a smooth curve continuously depends on $\mathrm{t}$ and $\lim _{t \rightarrow 0} \partial \Omega_{t}=\partial \Omega_{0}$. Throughout this section we always assume the multiplicity of $\lambda_{2}\left(\Omega_{0}\right)$ is three. And sometimes $\lambda_{2}\left(\Omega_{0}\right)$ is written as $\lambda_{2} ; \lambda_{2}\left(\Omega_{t}\right)$ as $\lambda_{2}(t)$.

In this paper we are only interested in the domain variation where the second eigenvalue remains unchanged. This is possible because of the monotonicity and continuity of eigenvalues. 
Now, we consider a special kind of domain variations. Suppose the origin of the coordinate is at $p \in \partial \Omega, \mathrm{x}$-direction is in the tangent direction and $\mathrm{y}$-direction is in the interior normal direction. Let $\Gamma$ be the part of boundary which can be written as

$$
\Gamma=(x, l(x)) \quad-b \leq x \leq b .
$$

And let $\Gamma^{1}=(x, l(x)),-b \leq x \leq 0, \quad \Gamma^{2}=(x, l(x)), 0 \leq x \leq b$. Let $h(x) \in$ $C_{0}^{\infty}(0,1), h(x) \geq 0$ and $h(x) \not \equiv 0$ in $(0,1)$.

Put

$$
\begin{gathered}
\Gamma_{t}^{1}=\left\{\left(x, l(x)+\beta(t) h\left(-\frac{x}{t}\right)\right), \quad-b t \leq x \leq 0\right\}, \\
\Gamma_{t}^{2}=\left\{\left(x, l(x)-\alpha(t) h\left(\frac{x}{t}\right)\right), \quad 0 \leq x \leq b t\right\},
\end{gathered}
$$

where $\beta(t)>0, \alpha(t)>0$ are to be determined for $0<t<\delta$.

We consider the domain variation where $\Gamma$ is replaced by $\Gamma_{t}^{1}$ and $\Gamma_{t}^{2}$. And at present, we assume $\alpha(t), \beta(t)$ are chosen so that $\lambda_{2}(t)=\lambda_{2}$. Moreover $\alpha(t) \rightarrow 0, \quad$ as $t \rightarrow 0 ; \beta(t) \rightarrow 0, \quad$ as $t \rightarrow 0$.

Suppose $\lambda_{1}(t)<\lambda_{2}(t) \leq \lambda_{3}(t) \leq \lambda_{4}(t)$ be the first four eigenvalues of $\Omega_{t}$. Let $\phi_{1}(t), \phi_{2}(t), \phi_{3}(t), \phi_{4}(t)$ be the corresponding orthogonal eigenfunctions. That is

$$
\int_{\Omega_{t}} \phi_{i}(t) \phi_{j}(t) d x d y=\delta_{i j}, \quad 1 \leq i, j \leq 4
$$

where $\phi_{i}(t)$ is a function of $(x, y)$ and $t$ is the parameter. Since we assumed that the multiplicity of $\Omega_{0}$ is three, then

$$
\begin{aligned}
& \lambda_{3}(t) \longrightarrow \lambda_{2}, \text { as } t \rightarrow 0 . \\
& \lambda_{4}(t) \longrightarrow \lambda_{2}, \text { as } t \rightarrow 0 .
\end{aligned}
$$

We may assume for convenience that as $t \rightarrow 0$

$$
\begin{aligned}
& \phi_{2}(t) \longrightarrow \phi_{2}, \\
& \phi_{3}(t) \longrightarrow \phi_{3}, \\
& \phi_{4}(t) \longrightarrow \phi_{4},
\end{aligned}
$$

otherwise we choose a sequence of $t$, since our proof is still true for a sequence of $\left\{t_{n}\right\}$ with $t_{n} \rightarrow 0$. 
Lemma 3.1. The nodal line $N\left(\phi_{2}(t)\right)$ intersects the boundary at $\Gamma_{t}$ exactly twice with the second order intersection or once with the third order intersection for small $t$.

Here the second order intersection means that the intersection point is a second order vanishing point.

Proof. We prove the lemma by contradictions.

First suppose the nodal line $N\left(\phi_{2}(t)\right)$ does not intersect $\Gamma_{t}$ at all. Let q be a point on the boundary which is not in $\Gamma_{t}$ and connected in $\partial \Omega_{t}$ with the origin p. Now, consider domain $\Omega_{0}$, through points p,q by lemma 2.3, we know that there exists a second eigenfunction $\phi$ such that $N(\phi)$ intersects $\partial \Omega$ at points p,q. Moreover, by Courant nodal domain theorem, we know that $\frac{\partial \phi}{\partial n}$ is positive on one of $\widehat{p q}$ and negative on the other $\widehat{p q}$. In particular, $\frac{\partial \phi}{\partial n}$ has different signs on $\Gamma^{1}$ and $\Gamma^{2}$. And $\frac{\partial \phi}{\partial n}$ on $\Gamma^{2}$ and $\phi$ on $\Gamma_{t}^{1}$ have the same sign as t sufficiently small.

Since $\phi$ satisfies $(1.1)$ and $\phi_{2}(t)$ satisfies

$$
\left\{\begin{array}{l}
\Delta \phi_{2}(t)+\lambda_{2} \phi_{2}(t)=0 \quad \text { in } \quad \Omega_{t} \\
\left.\phi_{2}(t)\right|_{\partial \Omega_{t}}=0 .
\end{array}\right.
$$

We can deduce from (1.1) and (3.10)

$$
\int_{\partial\left(\Omega_{t} \cap \Omega\right)}\left(\phi \frac{\phi_{2}(t)}{\partial n}-\phi_{2}(t) \frac{\partial \phi}{\partial n}\right)=0 .
$$

That is

$$
\int_{\Gamma_{t}^{1}} \phi \frac{\phi_{2}(t)}{\partial n}-\int_{\Gamma^{2}} \phi_{2}(t) \frac{\partial \phi}{\partial n}=0
$$

But $\phi_{2}(t)$ on $\Gamma^{2}$ on and $\frac{\partial \phi_{2}(t)}{\partial n}$ on $\Gamma_{t}^{1}$ have different sign here. Then as t small (3.12) is impossible.

Now, suppose $N\left(\phi_{2}(t)\right)$ intersects $\Gamma_{t}$ only once with a second order intersection. We may assume this intersection point is $p_{t}$. If $p_{t}$ is in $\Gamma_{t}^{2}$, we assume as t sufficiently small, $N\left(\phi_{2}(t)\right)$ intersects $\Gamma$ at $p_{t}^{*}$ and exactly once with the second order intersection. Otherwise $\phi_{2}$ the limitation of $\phi_{2}(t)$ would have at least fourth order vanishing at $\mathrm{p}$ which is impossible.

Again we consider the domain $\Omega_{0}$. By lemma 2.3 and remark 2.4, we know that there exists an eigenfunction $\phi$, such that $N(\phi)$ intersects the boundary of $\Omega$ at points $\mathrm{p}, p_{t}$ or $\mathrm{p}, p_{t}^{*}$ in the second case. Since $\frac{\partial \phi}{\partial n}$ has different signs on $\Gamma^{1}$ and $\Gamma^{2}$, then $N(\phi)$ does not intersect $\Gamma_{t}^{1}$ as t sufficiently 
small. Compare (1.1) with (3.10) we can also obtain (3.12). In this case, we can deduce also, by our choice of $\phi$ that $\phi \frac{\partial \phi_{2}(t)}{\partial n}$ on $\Gamma_{t}^{1}$ and $\phi_{2}(t) \frac{\partial \phi}{\partial n}$ on $\Gamma^{2}$ have different signs. We again reach a contradiction.

Corollary 3.2. The multiplicity of $\lambda_{2}(t)$ on the domain $\Omega_{t}$ is one.

Proof. Suppose the multiplicity of $\lambda_{2}(t)$ on $\Omega_{t}$ is not one. Let $\phi_{2}(t), \phi_{3}(t)$ be orthogonal eigenfunctions corresponding to $\lambda_{2}(t)$. Let $\mathrm{q}$ be the point as in the proof of lemma 3.1. We can find a linear combination of $\phi_{2}(t)$ and $\phi_{3}(t)$, such that the nodal line of it intersects the boundary at point q. Then by remark 2.2, we know that its nodal line intersects $\Gamma_{t}$ at most once and with a second order intersection. This contradicts lemma 3.1.

Corollary 3.3. The nodal line $N\left(\phi_{2}(t)\right)$ can not intersect $\partial \Omega_{t}$ at point $p$.

The proof is similar to that of lemma 3.1.

Remark 3.4. The second eigenfunction $\phi_{2}$ which is the limitation of $\phi_{2}(t)$ has a third order vanishing at point $p$. And the $\phi_{2}$ is actually unique. This can be seen easily from remark 2.5 .

Now we turn to study the properties of $\phi_{3}$ and $\phi_{4}$.

Lemma 3.5. The nodal line $N\left(\phi_{3}\right)$ intersects the boundary at point $p$. Moreover it is a second order intersection.

Proof. Suppose that $N\left(\phi_{3}\right)$ does not intersect the boundary at point p. For t sufficiently small neither does $N\left(\phi_{3}(t)\right)$. And we may assume $\phi_{3}(t)$ near point $\mathrm{p}$ in $\Omega_{t}$ is positive, $\frac{\partial \phi_{4}}{\partial n}(t, p) \leq 0$.

We choose a constant $k=k(t) \geq 0$, such that

$$
\left.\frac{\partial}{\partial n}\left(\phi_{4}(t)-k(t) \phi_{3}(t)\right)\right|_{p}=0 .
$$

Now if $\frac{\partial}{\partial n}\left(\phi_{4}(t)-k(t) \phi_{3}(t)\right) \geq 0$ near $\mathrm{p}$ for a sequence of t's going to zero, then for the limit $\phi_{4}-k \phi_{3}$ we would have $\frac{\partial}{\partial n}\left(\phi_{4}-k \phi_{3}\right) \geq 0$ near $\mathrm{p}$ and is 0 at $\mathrm{p}$. So $\phi_{4}-k \phi_{3}$ would have a third order vanishing at $\mathrm{p}$, contradicting the fact that $\phi_{2}$ is the only second eigenfunction with this property. Similarly one shows that $\frac{\partial}{\partial n}\left(\phi_{4}(t)-k(t) \phi_{3}(t)\right)$ can not have more than one zeros near p. So (3.13) means that the nodal line $N\left(\phi_{4}(t)-k(t) \phi_{3}(t)\right)$ intersects the 
boundary $\partial \Omega_{t}$ at $\mathrm{p}$. Moreover, $N\left(\phi_{4}(t)-k(t) \phi_{3}(t)\right)$ intersects $\Gamma_{t}$ only at point $\mathrm{p}$ for $\mathrm{t}$ sufficiently small. We can deduce

$$
\left\{\begin{array}{l}
\Delta\left(\phi_{4}(t)-k(t) \phi_{3}(t)\right)+\lambda_{2}\left(\phi_{4}(t)-k(t) \phi_{3}(t)\right)=w(t) \quad \text { in } \quad \Omega_{t} \\
\left.\left(\phi_{4}(t)-k(t) \phi_{3}(t)\right)\right|_{\partial \Omega_{t}}=0
\end{array}\right.
$$

where $w(t)=\left(\lambda_{2}-\lambda_{4}(t)\right) \phi_{4}(t)-\left(\lambda_{2}-\lambda_{3}(t) k(t) \phi_{3}(t)\right.$. As $t \rightarrow 0$, let

$$
\frac{w(t)}{|w(t)|_{L^{\infty}\left(\Omega_{t}\right)}} \rightarrow w=\beta_{2} \phi_{4}-\beta_{1} \phi_{3}
$$

where $\beta_{1}, \beta_{2}$ are constants. Since $\lambda_{4}(t) \geq \lambda_{3}(t)>\lambda_{2}$, then $\beta_{1}, \beta_{2} \leq 0$ and at least one of them is not zero. In particular $\beta_{2} \neq 0$.

We can check easily that $\phi_{2}, \beta_{2} \phi_{4}-\beta_{1} \phi_{3}$ and $\beta_{1} \phi_{4}+\beta_{2} \phi_{3}$ are orthogonal eigenfunctions corresponding to $\lambda_{2}$ in $\Omega$. Moreover

$$
\left.\frac{\partial}{\partial n}\left(\beta_{2} \phi_{3}+\beta_{1} \phi_{4}\right)\right|_{p}>0
$$

from our assumption.

We claim that the nodal line $N\left(\phi_{2}+\beta_{2} \phi_{3}+\beta_{1} \phi_{4}\right)$ does not intersect $\partial \Omega$ near point $\mathrm{p}$. In fact, $\phi_{2}$ has a third vanishing order at point $\mathrm{p}$. From the expansion (2.11), we can assume $\frac{\partial \phi_{2}}{\partial n} \geq 0$ on the boundary near p. Then $\phi_{2}+\beta_{2} \phi_{3}+\beta_{1} \phi_{4}$ have our desired property.

Notice that $\phi_{2}+\beta_{2} \phi_{3}+\beta_{1} \phi_{4}$ and $w$ are orthogonal, then we can choose $c(t)$ such that

$$
\int_{\Omega \cap \Omega_{t}}\left[\phi_{2}+\beta_{2} \phi_{3}+\beta_{1} \phi_{4}+c(t)\left(\beta_{2} \phi_{4}-\beta_{1} \phi_{3}\right)\right] \frac{w(t)}{|w(t)|_{L^{\infty}\left(\Omega_{t}\right)}}=0,
$$

and $c(t) \rightarrow 0$, as $t \rightarrow 0$. In fact, as $t \rightarrow 0$

$$
\int_{\Omega \cap \Omega_{t}}\left(\beta_{2} \phi_{4}-\beta_{1} \phi_{3}\right) \frac{w(t)}{|w(t)|_{L^{\infty}\left(\Omega_{t}\right)}} \rightarrow \int_{\Omega} w^{2}>0
$$

$$
\int_{\Omega \cap \Omega_{t}}\left(\phi_{2}+\beta_{2} \phi_{3}+\beta_{1} \phi_{4}\right) \frac{w(t)}{|w(t)|_{L^{\infty}\left(\Omega_{t}\right)}} \longrightarrow 0
$$

Set

$$
\phi=\phi_{2}+\beta_{2} \phi_{3}+\beta_{1} \phi_{4}+c(t)\left(\beta_{2} \phi_{4}-\beta_{1} \phi_{3}\right) .
$$


We know $\phi$ is also an eigenfunction corresponding to $\lambda_{2}$ and $N(\phi)$ does not intersect $\partial \Omega$ near point $\mathrm{p}$ as $\mathrm{t}$ small. Now we compare $\phi$ with $\phi_{4}(t)-$ $k(t) \phi_{3}(t)$, as in the proof of lemma 3.1 , we have

$$
\int_{\Gamma_{t}^{1}} \phi \frac{\partial}{\partial n}\left(\phi_{4}(t)-k(t) \phi_{3}(t)\right)-\int_{\Gamma^{2}} \frac{\partial \phi}{\partial n}\left(\phi_{4}(t)-k(t) \phi_{3}(t)\right)=0,
$$

which leads to a contradiction as t sufficiently small.

Remark 3.6. The $\phi_{3}$ which is the limitation of $\phi_{3}(t)$ is unique. Since it has a second vanishing order at $\mathrm{p}$ and are orthogonal with $\phi_{2}$ which has a third vanishing order at $\mathrm{p}$. So $\phi_{3}$ must be unique. And therefore $\phi_{4}$ which is the limitation of $\phi_{4}(t)$ is also unique. The vanishing order of $\phi_{4}$ at $\mathrm{p}$ is one.

Since $\phi_{2}, \phi_{3}, \phi_{4}$ are unique, then

$$
\begin{aligned}
& \lim _{t \rightarrow 0} \phi_{2}(t)=\phi_{2}, \\
& \lim _{t \rightarrow 0} \phi_{3}(t)=\phi_{3}, \\
& \lim _{t \rightarrow 0} \phi_{4}(t)=\phi_{4} .
\end{aligned}
$$

We actually do not need to take a sequence in the above proof.

In the following, we let $\phi(t, p)$ be the value of $\phi(t)$ at point $\mathrm{p}$.

Lemma 3.6. Assume $\alpha(t)=O\left(t^{5}\right)$ and $\beta(t)=O\left(t^{5}\right)$ as $t \rightarrow 0$ in the domain variation, then as $t \rightarrow 0$

$$
\frac{\partial^{2} \phi_{2}}{\partial y \partial x}(t, p)=O(t)
$$

$$
\frac{\partial \phi_{2}}{\partial y}(t, p)=O\left(t^{2}\right)
$$

Proof. By lemma 3.1, we know that there exist $p_{1}, p_{2} \in \Gamma_{t}$ and $p_{1}, p_{2} \in$ $N\left(\phi_{2}(t)\right)$. Then

$$
\frac{\partial \phi_{2}}{\partial n}\left(t, p_{1}\right)=\frac{\partial \phi_{2}}{\partial n}\left(t, p_{2}\right)=0 .
$$

Moreover $\frac{\partial^{2} \phi_{2}}{\partial n \partial T}\left(t, p_{1}\right)$ and $\frac{\partial^{2} \phi_{2}}{\partial n \partial T}\left(t, p_{2}\right)$ have different sign, if $p_{1}$ and $p_{2}$ are different, where $\mathrm{n}$ is the exterior normal direction and $\mathrm{T}$ is the tangent direction 
at the corresponding points. Then there always exists $p_{3}$ on $\Gamma_{t} \cap{ }_{p_{1} p_{2}}$, such that

$$
\frac{\partial^{2} \phi_{2}}{\partial n \partial T}\left(t, p_{3}\right)=0
$$

Note that on $\Gamma_{t}^{2}$ in the local chart

$$
\begin{aligned}
& \text { (3.26) } T=\left(\frac{1}{\sqrt{1+\left(l^{\prime}(x)+\frac{\alpha(t)}{t} h^{\prime}(x / t)\right)^{2}}},\right. \\
& \left.\frac{l^{\prime}(x)+\frac{\alpha(t)}{t} h^{\prime}(x / t)}{\sqrt{1+\left(l^{\prime}(x)+\frac{\alpha(t)}{t} h^{\prime}(x / t)\right)^{2}}}\right)=\left(T_{x}, T_{y}\right) \\
& \text { (3.27) } n=\left(\frac{l^{\prime}(x)+\frac{\alpha(t)}{t} h^{\prime}(x / t)}{\sqrt{1+\left(l^{\prime}(x)+\frac{\alpha(t)}{t} h^{\prime}(x / t)\right)^{2}}},\right. \\
& \left.-\frac{1}{\sqrt{1+\left(l^{\prime}(x)+\frac{\alpha(t)}{t} h^{\prime}(x / t)\right)^{2}}}\right)=\left(n_{x}, n_{y}\right)
\end{aligned}
$$

Then

$$
\frac{\partial \phi_{2}(t)}{\partial n}=\frac{\partial \phi_{2}(t)}{\partial x} n_{x}+\frac{\partial \phi_{2}(t)}{\partial y} n_{y}
$$

$$
\begin{aligned}
& \frac{\partial^{2} \phi_{2}(t)}{\partial_{T} \partial n}=\frac{\partial}{\partial x}\left(\frac{\partial \phi_{2}(t)}{\partial x} n_{x}+\frac{\partial \phi_{2}(t)}{\partial y} n_{y}\right) T_{x}+ \\
& \quad+\frac{\partial}{\partial y}\left(\frac{\partial \phi_{2}(t)}{\partial x} n_{x}+\frac{\partial \phi_{2}(t)}{\partial y} n_{y}\right) T_{y}
\end{aligned}
$$

For all $q \in \Gamma_{t}$ we have

$$
\frac{\partial^{2} \phi_{2}(t)}{\partial_{T} \partial n}=-\frac{\partial^{2} \phi_{2}(t)}{\partial_{x} \partial y}+O(t)
$$

In particular at $p_{3}$,

$$
\frac{\partial^{2} \phi_{2}}{\partial_{x} \partial y}\left(t, p_{3}\right)=O(t)
$$


From the regularity of elliptic equations, we know that $\phi_{2} \in C^{3}$. Then

$$
\frac{\partial^{2} \phi_{2}}{\partial_{x} \partial y}(t, p)=O(t)
$$

In fact, (3.31) is true for all points of $\Gamma_{t}$.

For the proof of (3.23), we observe that

$$
\nabla \phi_{2}\left(t, p_{1}\right)=\nabla \phi_{2}\left(t, p_{2}\right)=0,
$$

together with (3.31) for some q on $\Gamma_{t}$ we obtain the proof.

\section{Proof of the Theorem.}

In this section, we use the same notations as in section 3 . We first specify the function $\alpha(t), \beta(t)$ which given in (3.2) and (3.3).

Let $\beta_{0}(t)=t^{m}$ and $\alpha_{0}(t, m)$ be chosen so that $\lambda_{2}(t)=\lambda_{2}$. This is possible as we mentioned before. Notice that for each $1>t>0, \alpha_{0}(t, m)$ is a monotone decreasing function of $\mathrm{m}$, and

$$
\lim _{m \rightarrow \infty} \alpha_{0}(t, m)=0 \text {. }
$$

Choose $m=m(t)$ be a smooth function for small $\mathrm{t}$, and

$$
\begin{array}{ll}
1^{0} & \beta(t)=t^{m(t)}=O\left(t^{5}\right) \quad \text { as } \quad t \quad \rightarrow 0 \\
2^{0} & \alpha(t)=\alpha_{0}(t, m(t))<t^{5} \quad \text { for } t \quad \text { small. }
\end{array}
$$

It is easy to check that domain $\Omega_{t}$ is uniformly $C^{4}$. And then as $t \rightarrow 0$

$$
\phi_{2}(t) \longrightarrow \phi_{2} \quad \text { in } \quad C^{3}\left(\Omega_{t} \cap \Omega\right) .
$$

Now we give the following lemmas.

Lemma 4.1. Under the above notations and assumptions, the limit points of $\frac{\alpha(t)}{\beta(t)}$ are $1, k_{0}$ and $\frac{1}{k_{0}}$ for some constant $1>k_{0}>0$ as $t \rightarrow 0$, therefore $\alpha(t)$ and $\beta(t)$ have the same order as $t \rightarrow 0$.

Proof. Compare $\phi_{2}(t)$ with $\phi_{i}, i=2,3,4$ as in the proof of lemma 3.1, we have

$$
\int_{\Gamma_{t}^{1}} \frac{\partial \phi_{2}}{\partial n}(t) \phi_{i}=\int_{\Gamma^{2}} \frac{\partial \phi_{i}}{\partial n} \phi_{2}(t)
$$


where $i=2,3,4$.

Now we calculate the value of the two terms in (4.2).

$$
\int_{\Gamma_{t}^{1}} \frac{\partial \phi_{2}}{\partial n}(t) \phi_{i}=\left.\int_{-b t}^{0}\left(\frac{\partial \phi_{2}}{\partial n}(t) \phi_{i}\right)\right|_{\Gamma_{t}^{1}} \sqrt{1+\left(l^{\prime}(x)+\frac{\beta(t)}{t} h^{\prime}(-x / t)\right)^{2}} d x
$$

Since

$$
\phi_{i}(x, l(x)+\beta(t) h(-x / t))=\frac{\partial \phi_{i}}{\partial y}(x, l(x)) h(-x / t) \beta(t)+O\left(t^{5}\right)
$$

then

$$
\begin{aligned}
& \int_{\Gamma_{t}^{1}} \frac{\partial \phi_{2}}{\partial n}(t) \phi_{i}= \\
& \int_{-b t}^{0} \frac{\partial \phi_{2}}{\partial n}\left(t, x, l(x)+\beta(t) h\left(-\frac{x}{t}\right)\left[\frac{\partial \phi_{i}}{\partial y}(x, l(x)) h\left(-\frac{x}{t}\right) \beta(t)+O\left(t^{5}\right)\right] L(x) d x,\right.
\end{aligned}
$$

where $L(x)=\sqrt{1+\left(l^{\prime}(x)+\beta(t) / t h^{\prime}\left(-\frac{x}{t}\right)\right)^{2}}$. For convenience we assume $b=$ 1 in the following.

By Taylor expansion at the origin in $\Gamma_{t}$, we have

$$
\begin{gathered}
\phi_{2}(t)=a_{0}(t) y+a_{1}(t) x y+a_{2}(t)\left(x^{2}-y^{2}\right)+a_{3}(t) y x^{2}+ \\
a_{4}(t) x^{3}+a_{5}(t) y^{3}+a_{6}(t) y^{2} x+O\left(t^{4}\right),
\end{gathered}
$$

where we used the fact that for $t>0$, the vanishing order of $\phi_{2}(t)$ is finite at p.(see Melas [8]). And the first two terms are harmonic polynomials. All $a_{i}(t)$ are continuous functions. If $\phi_{2}(t)$ has a third order vanishing at $p$, by remark 2.5 we know $a_{3}(t)$ is not zero.

Recall (3.27), we can deduce

$$
\begin{aligned}
& \text { (4.7) }\left.\quad \frac{\partial}{\partial n} \phi_{2}(t)\right|_{\Gamma_{t}^{1}}= \\
& =\left[a_{0}(t)+a_{1}(t) x-2 a_{2}(t) y+a_{3}(t) x^{2}\right] n_{y}+\left[a_{1}(t) y+2 a_{2}(t) x\right] n_{x}+O\left(t^{3}\right),
\end{aligned}
$$

where $y=l(x)+\beta(t) h(-x / t), n_{x}=O(t)$ and $n_{y}=1+O\left(t^{2}\right)$ in $\Gamma_{1}^{t}$.

Put $x=t s$, then

$$
\left.\frac{\partial \phi_{2}}{\partial n}(t)\right|_{\Gamma_{t}^{1}}=a_{0}(t)+a_{1}(t) t s+a_{3}(t) t^{2} s^{2}+o\left(t^{2}\right) .
$$

Similarly we can deduce for $0 \leq x \leq t$

$$
\begin{aligned}
\frac{\partial \phi_{i}}{\partial y}(x, l(x))=\frac{\partial \phi_{i}}{\partial y}(p) & +\frac{\partial^{2} \phi_{i}}{\partial x \partial y}(p) t s+ \\
& +\left[\frac{1}{2} \frac{\partial^{3} \phi_{i}}{\partial x^{2} \partial y}(p)+\frac{\partial^{2} \phi_{i}}{\partial y^{2}}(p) l^{\prime \prime}(0)\right] t^{2} s^{2}+O\left(t^{3}\right)
\end{aligned}
$$


Substitute (4.8),(4.9) into (4.5) we obtain

$$
\begin{aligned}
\int_{\Gamma_{t}^{1}} \frac{\partial \phi_{2}}{\partial n}(t) \phi_{i} & =\left(1+O\left(t^{2}\right)\right) \beta(t) t \int_{-1}^{0}\left[a_{0}(t)+a_{1}(t) t s+a_{3}(t) t^{2} s^{2}+o\left(t^{2}\right)\right] \\
& {\left[\frac{\partial \phi_{i}}{\partial y}(p)+\frac{\partial^{2} \phi_{i}}{\partial x \partial y}(p) t s+\left(\frac{\partial^{2} \phi_{i}}{\partial y^{2}}(p) l^{\prime \prime}(0)+\frac{1}{2} \frac{\partial^{3} \phi_{i}}{\partial x^{2} \partial y}(p)\right) t^{2} s^{2}+\right.} \\
& \left.O\left(t^{3}\right)\right] h(-s) d s .
\end{aligned}
$$

Since $\phi_{2}$ has a third vanishing order at $\mathrm{p}$ and $\phi_{3}$ has a second order vanishing at $\mathrm{p}$, we have for $i=2,3,4$,

$$
\begin{array}{r}
\int_{\Gamma_{t}^{1}} \frac{\partial \phi_{2}}{\partial n}(t) \phi_{2}=\beta(t) t^{3} \int_{-1}^{0}\left[a_{0}(t)+a_{1}(t) t s+a_{3}(t) t^{2} s^{2}+o\left(t^{2}\right)\right] \\
{\left[1 / 2 \frac{\partial^{3} \phi_{2}}{\partial x^{2} \partial y}(p) s^{2}+O(t)\right] h(-s) d s\left[1+O\left(t^{2}\right)\right]}
\end{array}
$$

$$
\begin{gathered}
\int_{\Gamma_{t}^{1}} \frac{\partial \phi_{2}}{\partial n}(t) \phi_{3}=\beta(t) t^{2} \int_{-1}^{0}\left[a_{0}(t)+a_{1}(t) t s+a_{3}(t) t^{2} s^{2}+o\left(t^{2}\right)\right] \\
{\left[\frac{\partial^{2} \phi_{3}}{\partial x \partial y}(p) s+O(t)\right] h(-s) d s\left[1+O\left(t^{2}\right)\right] .}
\end{gathered}
$$

$$
\begin{gathered}
\int_{\Gamma_{t}^{1}} \frac{\partial \phi_{2}}{\partial n}(t) \phi_{4}=\beta(t) t \int_{-1}^{0}\left[a_{0}(t)+a_{1}(t) t s+a_{3}(t) t^{2} s^{2}+o\left(t^{2}\right)\right] \\
{\left[\frac{\partial \phi_{4}}{\partial y}(p)+O(t)\right] h(-s) d s\left[1+O\left(t^{2}\right)\right]}
\end{gathered}
$$

where $a_{0}(t)=O\left(t^{2}\right), a_{1}(t)=O(t)$ and $a_{3}(t) \rightarrow \frac{1}{2} \frac{\partial^{3} \phi_{2}}{\partial x^{2} \partial y}(p) \neq 0$, because of lemma 3.8.

Again on $\Gamma^{2}$ we can deduce

$$
\begin{gathered}
\int_{\Gamma^{2}} \frac{\partial \phi_{4}}{\partial n} \phi_{2}(t)=\alpha(t) t \int_{0}^{1}\left[a_{0}(t)+a_{1}(t) t s+a_{3}(t) t^{2} s^{2}+o\left(t^{2}\right)\right] \\
{\left[\frac{\partial \phi_{4}}{\partial y}(p)+O(t)\right] h(s) d s\left[1+O\left(t^{2}\right)\right]}
\end{gathered}
$$


(4.15)

$$
\begin{gathered}
\int_{\Gamma^{2}} \frac{\partial \phi_{3}}{\partial n} \phi_{2}(t)=\alpha(t) t^{2} \int_{0}^{1}\left[a_{0}(t)+a_{1}(t) t s+a_{3}(t) t^{2} s^{2}+o\left(t^{2}\right)\right] \\
{\left[\frac{\partial^{2} \phi_{3}}{\partial x \partial y}(p) s+O(t)\right] h(s) d s\left[1+O\left(t^{2}\right)\right]}
\end{gathered}
$$

(4.16)

$$
\begin{gathered}
\int_{\Gamma^{2}} \frac{\partial \phi_{2}}{\partial n} \phi_{2}(t)=\alpha(t) t^{3} \int_{0}^{1}\left[a_{0}(t)+a_{1}(t) t s+a_{3}(t) t^{2} s^{2}+o\left(t^{2}\right)\right] \\
{\left[\frac{\partial^{3} \phi_{2}}{\partial x^{2} \partial y}(p) s^{2}+O(t)\right] h(-s) d s\left[1+O\left(t^{2}\right)\right] .}
\end{gathered}
$$

For some sequence $\left\{t_{i}\right\}, t_{i} \rightarrow 0$, suppose

$$
\frac{a_{0}\left(t_{i}\right)}{t_{i}^{2}} \longrightarrow x
$$

$$
\frac{a_{1}\left(t_{i}\right)}{t_{i}} \longrightarrow y
$$

$$
a_{3}\left(t_{i}\right) \longrightarrow z
$$

Then we have

$$
\begin{aligned}
\lim _{t_{i} \rightarrow 0} \frac{\beta\left(t_{i}\right)}{\alpha\left(t_{i}\right)} & =\frac{\int_{0}^{1}\left(x s+y s^{2}+z s^{3}\right) h(s) d s}{\int_{-1}^{0}\left(x s+y s^{2}+z s^{3}\right) h(-s) d s} \\
& =\frac{\int_{0}^{1}\left(x+y s+z s^{2}\right) h(s) d s}{\int_{-1}^{0}\left(x+y s+z s^{2}\right) h(-s) d s} \\
& =\frac{\int_{0}^{1}\left(x s^{2}+y s^{3}+z s^{4}\right) h(s) d s}{\int_{-1}^{0}\left(x s^{2}+y s^{3}+z s^{4}\right) h(-s) d s} \\
& =k \geq 0 .
\end{aligned}
$$

Set $b_{i}=\int_{0}^{1} s^{i} h(s) d s, i=0,1,2,3,4$. We can deduce from (4.20)

$$
\left\{\begin{array}{l}
(k-1) b_{0} x-(k+1) b_{1} y+(k-1) b_{2} z=0 \\
(k+1) b_{1} x-(k-1) b_{2} y+(k+1) b_{3} z=0 \\
(k-1) b_{2} x-(k+1) b_{3} y+(k-1) b_{4} z=0
\end{array}\right.
$$


Since $x, y, z$ are not all zero, then the determinant of the coefficient is zero. We have

$$
D=\left|\begin{array}{lll}
(k-1) b_{0} & -(k+1) b_{1} & (k-1) b_{2} \\
(k+1) b_{1} & -(k-1) b_{2} & (k+1) b_{3} \\
(k-1) b_{2} & -(k+1) b_{3} & (k-1) b_{4}
\end{array}\right|=0
$$

A simple calculation shows that

$$
\begin{aligned}
D= & \frac{k-1}{b_{3}}\left[(k-1)^{2}\left(b_{2} b_{4}\left(b_{1} b_{2}-b_{0} b_{3}\right)-b_{2}^{2}\left(b_{1} b_{4}-b_{2} b_{3}\right)\right)-\right. \\
& \left.(k+1)^{2}\left(b_{3}^{2}\left(b_{1} b_{2}-b_{0} b_{3}\right)-b_{1} b_{3}\left(b_{1} b_{4}-b_{2} b_{3}\right)\right)\right] .
\end{aligned}
$$

Put

$$
\begin{aligned}
& A=b_{2} b_{4}\left(b_{1} b_{2}-b_{0} b_{3}\right)-b_{2}^{2}\left(b_{1} b_{4}-b_{2} b_{3}\right) \\
& B=b_{3}^{2}\left(b_{1} b_{2}-b_{0} b_{3}\right)-b_{1} b_{3}\left(b_{1} b_{4}-b_{2} b_{3}\right) .
\end{aligned}
$$

From Holder inequality we can check $b_{1} b_{2}<b_{0} b_{3}$ and $b_{2} b_{3}<b_{1} b_{4}$. That is $A<0$ and $B<0$.

Then we know either $k=1$ or

$$
k^{2}-2 \frac{A+B}{A-B} k+1=0 .
$$

We can show that $A<B$. (see appendix) That is (4.24) always has two positive solutions. Then we finished the proof of lemma.

Lemma 4.2. Under the assumptions of lemma 4.1, we have

$$
\lim _{t \rightarrow 0} \frac{\alpha(t)}{\beta(t)}=1
$$

Proof. Suppose $\lim _{t \rightarrow 0} \frac{\alpha(t)}{\beta(t)}=k_{0}, k_{0}$ is a solution of (4.24). We will use the fact that $\lambda_{2} \leq \lambda_{3}(t) \leq \lambda_{4}(t)$ to rule out this possibility.

Now compare $\phi_{4}(t)$ with $\phi_{4}$, we have

$$
\left(\lambda_{4}(t)-\lambda_{2}\right) \int_{\Omega \cap \Omega_{t}} \phi_{4}(t) \phi_{4}=\int_{\Gamma^{2}} \frac{\partial \phi_{4}}{\partial n} \phi_{4}(t)-\int_{\Gamma_{t}^{1}} \frac{\partial \phi_{4}(t)}{\partial n} \phi_{4} .
$$

We can calculate the right hand sides of (4.25) as in lemma 4.1, we deduce

$$
\begin{aligned}
& \left(\lambda_{2}-\lambda_{4}(t)\right) \int_{\Omega \cap \Omega_{t}} \phi_{4}(t) \phi_{4}= \\
& \quad=t(\alpha(t)-\beta(t)) \frac{\partial \phi_{4}}{\partial y}(p) \frac{\partial \phi_{4}}{\partial y}(t, p) \int_{0}^{1} h(s) d s+O\left(\alpha(t) t^{2}\right) .
\end{aligned}
$$


Since $\frac{\partial \phi_{4}}{\partial y}(t, p) \rightarrow \frac{\partial \phi_{4}}{\partial y}(p)$ and $\lambda_{4}(t) \geq \lambda_{2}$, we deduce

$$
\alpha(t) \leq \beta(t),
$$

for $t \geq 0$ and sufficiently small.

Now compare $\phi_{3}(t)$ with $\phi_{3}$ we have

$$
\begin{aligned}
\left(\lambda_{2}-\lambda_{3}(t)\right) & \int_{\Omega \cap \Omega_{t}} \phi_{3}(t) \phi_{3}=\frac{\partial \phi_{3}}{\partial y}(t, p) \frac{\partial^{2} \phi_{3}}{\partial y \partial x}(p) \int s h(s) d s(\alpha(t)+\beta(t)) t^{2} \\
& +\frac{\partial^{2} \phi_{3}}{\partial y \partial x}(t, p) \frac{\partial^{2} \phi_{3}}{\partial x \partial y}(p) \int s^{2} h(s) d s(\alpha(t)-\beta(t)) t^{3}+o\left(\alpha(t) t^{3}\right)
\end{aligned}
$$

here we used the fact that $\frac{\partial \phi_{3}}{\partial y}(p)=0$.

First from (4.27) we know that

$$
\lambda_{2}-\lambda_{3}(t)=o\left(\alpha(t) t^{2}\right) .
$$

Compare $\phi_{3}(t)$ with $\phi_{4}$ we have

$$
\begin{aligned}
\left(\lambda_{2}-\lambda_{3}(t)\right) \int_{\Omega \cap \Omega_{t}} \phi_{3}(t) \phi_{4}= & \frac{\partial \phi_{3}}{\partial y}(t, p) \frac{\partial \phi_{4}}{\partial y}(p) \int h(s) d s(\alpha(t)-\beta(t)) t \\
& +\frac{\partial^{2} \phi_{3}}{\partial y \partial x}(t, p) \frac{\partial \phi_{4}}{\partial y}(p) \int \operatorname{sh}(s) d s(\alpha(t)+\beta(t)) t^{2} \\
& +o\left(\alpha(t) t^{2}\right) .
\end{aligned}
$$

From (4.29), (4.28) we have

$$
\frac{\partial \phi_{3}}{\partial y}(t, p)=-\frac{\partial^{2} \phi_{3}}{\partial y \partial x}(t, p) \frac{\int s h(s) d s(\alpha(t)+\beta(t)) t}{\int h(s) d s(\alpha(t)-\beta(t))}+o(t),
$$

where $\frac{\partial^{2} \phi_{3}}{\partial y \partial x}(t, p) \longrightarrow \frac{\partial^{2} \phi_{3}}{\partial y \partial x}(p)$.

Substitute (4.30) into (4.27) and notice $\lambda_{2} \leq \lambda_{3}(t)$ we have

$$
\begin{aligned}
0 \geq & -\frac{\partial^{2} \phi_{3}}{\partial y \partial x}(t, p) \frac{\partial^{2} \phi_{3}}{\partial y \partial x}(p) \frac{\left(\int s h(s) d s\right)^{2}(\alpha(t)+\beta(t))^{2}}{\int h(s) d s(\alpha(t)-\beta(t))} \\
& +\frac{\partial^{2} \phi_{3}}{\partial y \partial x}(t, p) \frac{\partial^{2} \phi_{3}}{\partial y \partial x}(p) \int s^{2} h(s) d s(\alpha(t)-\beta(t))+o(\alpha(t)) .
\end{aligned}
$$


Recall (4.26) and the notation of $b_{i}, \mathrm{~A}$ and $\mathrm{B}$, we have

$$
0 \leq-\left(1+k_{0}\right)^{2} \frac{b_{1}^{2}}{b_{0}}+\left(1-k_{0}\right)^{2} b_{2} .
$$

Recall (4.24) then we have

$$
b_{0} b_{2} B \geq A b_{1}^{2}
$$

That is

$$
\begin{gathered}
b_{0} b_{2}\left[b_{3}^{2}\left(b_{1} b_{2}-b_{0} b_{3}\right)-b_{1} b_{3}\left(b_{1} b_{4}-b_{2} b_{3}\right)\right] \geq b_{1}^{2}\left(b_{2}^{3} b_{3}-b_{0} b_{2} b_{3} b_{4}\right) \\
\left(b_{0} b_{3}-b_{1} b_{2}\right)^{2} \leq 0
\end{gathered}
$$

This is a contradiction, since $b_{0} b_{3}>b_{1} b_{2}$. Then we proved the lemma.

Now suppose the limitation of $\frac{\alpha(t)}{\beta(t)}$ is one as $t \rightarrow 0$, we distinct the following two cases
(i) $\underline{\lim }_{t \rightarrow 0} \frac{\alpha(t) t}{\alpha(t)-\beta(t)}=c \neq 0$,
(ii) $\varliminf_{t \rightarrow 0} \frac{\alpha(t) t}{\alpha(t)-\beta(t)}=0$,

where c could be infinity.

We first prove that case (i) cannot occur.

Lemma 4.3. Under the assumptions of lemma 4.1, we have

$$
\lim _{t \rightarrow 0} \frac{\alpha(t) t}{\alpha(t)-\beta(t)}=0
$$

Proof. Suppose case (i) occur. Notice (4.25), we have as in the proof of lemma 4.2,

$$
\begin{aligned}
&\left(\lambda_{2}-\lambda_{4}(t)\right) \int_{\Omega \cap \Omega_{t}} \phi_{4}(t) \phi_{4}= t(\alpha(t)-\beta(t))\left(\frac{\partial \phi_{4}}{\partial y}(p) \frac{\partial \phi_{4}}{\partial y}(t, p)\right) \int_{0}^{1} h(s) d s \\
&+(\alpha(t)+\beta(t)) t^{2}\left[\frac{\partial^{2} \phi_{4}}{\partial x \partial y}(t, p) \frac{\partial \phi_{4}}{\partial y}(p)+\right. \\
&\left.\frac{\partial \phi_{4}}{\partial y}(t, p) \frac{\partial^{2} \phi_{4}}{\partial x \partial y}(p)\right] \int_{0}^{1} h(s) s d s+o\left(\alpha(t) t^{2}\right) .
\end{aligned}
$$


where $\frac{\partial \phi_{4}}{\partial y}(p) \neq 0$, and $\frac{\partial \phi_{4}}{\partial y}(t, p) \longrightarrow \frac{\partial \phi_{4}}{\partial y}(p), \frac{\partial^{2} \phi_{4}}{\partial x \partial y}(t, p) \longrightarrow \frac{\partial^{2} \phi_{4}}{\partial x \partial y}(p)$ and $\int_{\Omega \cap \Omega_{t}} \phi_{4}(t) \phi_{4} \rightarrow 1$ as $t \rightarrow 0$.

Similarly, compare $\phi_{4}(t)$ with $\phi_{3}$, we have

$$
\begin{aligned}
& \left(\lambda_{2}-\lambda_{4}(t)\right) \int_{\Omega \cap \Omega_{t}} \phi_{4}(t) \phi_{3}= \\
& \quad=(\alpha(t)+\beta(t)) t^{2} \frac{\partial \phi_{4}}{\partial y}(t, p) \frac{\partial^{2} \phi_{3}}{\partial x \partial y}(p) \int h(s) s d s+o\left(\alpha(t) t^{2}\right) .
\end{aligned}
$$

Here we again used the fact that $\frac{\partial \phi_{3}}{\partial y}(p)=0$.

Then from (4.36) and (4.37) we obtain

$$
\begin{aligned}
\frac{\partial \phi_{4}}{\partial y}(t, p) & \frac{\partial^{2} \phi_{3}}{\partial x \partial y}(p) \int h(s) s d s=\lim _{t \rightarrow 0} \int_{\Omega \cap \Omega_{t}} \phi_{4}(t) \phi_{3} \\
& \times\left[\frac{\partial \phi_{4}}{\partial y}(p) \frac{\partial^{2} \phi_{4}}{\partial x \partial y}(p) \int s h(s) d s+\frac{1}{2 c}\left(\frac{\partial \phi_{4}}{\partial y}\right)^{2} \int h(s) d s\right]
\end{aligned}
$$

where

$$
c=\lim _{t \rightarrow 0} \frac{\alpha(t) t}{\alpha(t)-\beta(t)} \neq 0 .
$$

Notice that $\frac{\partial \phi_{4}}{\partial y}(p) \neq 0$ and $\frac{\partial^{2} \phi_{3}}{\partial y \partial x}(p) \neq 0$, but $\lim _{t \rightarrow 0} \int_{\Omega \cap \Omega_{t}} \phi_{4}(t) \phi_{3}=0$ since $\phi_{4}(t) \rightarrow \phi_{4}$. Then we reach a contradiction. This is the proof of the lemma.

Lemma 4.4. Under the assumptions of lemma 4.1, as $t \rightarrow 0$ we have

$$
\begin{aligned}
& \phi_{2}(t)=c_{2}(t) \phi_{2}+c_{3}(t) \phi_{3}+c_{4}(t) \phi_{4}+o\left(t^{4}\right) \quad \text { in } \Omega \cap \Omega_{t} \\
& \phi_{3}(t)=d_{2}(t) \phi_{2}+d_{3}(t) \phi_{3}+d_{4}(t) \phi_{4}+o\left(t^{4}\right) \quad \text { in } \Omega \cap \Omega_{t} \\
& \phi_{4}(t)=e_{2}(t) \phi_{2}+e_{3}(t) \phi_{3}+e_{4}(t) \phi_{4}+o\left(t^{4}\right) \quad \text { in } \Omega \cap \Omega_{t},
\end{aligned}
$$

where $c_{i}(t)=\int_{\Omega \cap \Omega_{t}} \phi_{2}(t) \phi_{i}, d_{i}(t)=\int_{\Omega \cap \Omega_{t}} \phi_{3}(t) \phi_{i}$ and $e_{i}(t)=\int_{\Omega \cap \Omega_{t}} \phi_{4}(t) \phi_{i}$, $i=2,3,4$.

Proof. We only need to show (4.39), the remains can be proved similarly. Put

$$
w(t)=\phi_{2}(t)-c_{2}(t) \phi_{2}-c_{3}(t) \phi_{3}-c_{4}(t) \phi_{4}
$$


Then we know that $\mathrm{w}(\mathrm{t})$ are orthogonal to $\phi_{2}, \phi_{3}, \phi_{4}$ in the domain $\Omega \cap \Omega_{t}$.

Consider $\frac{w(t)}{t^{4}}$, it satisfies the equation of problem (1.1) in $\Omega \cap \Omega_{t}$. If there exists a sequence $\left\{t_{i}\right\}$ and $t_{i} \rightarrow 0$, such that

$$
\left|\frac{w\left(t_{i}\right)}{t_{i}^{4}}\right|_{L^{\infty}} \longrightarrow c \neq 0
$$

then $\left|\frac{w\left(t_{i}\right)}{t_{i}^{4}}\right|_{L^{\infty}}$ is archived in the interior of $\Omega \cap \Omega_{t}$, assume at $p_{i}$. We can suppose $p_{i} \rightarrow q \in \Omega$, because on the boundary $\partial\left(\Omega \cap \Omega_{t}\right), \frac{w\left(t_{i}\right)}{t_{i}^{4}} \longrightarrow 0$.

Consider $\left|\frac{w\left(t_{i}\right)}{t_{i}^{4}}\right|_{L^{\infty}}$, we know that $\mathrm{w}$ also satisfies (1.1) and $w \not \equiv 0$. Moreover, $\mathrm{w}$ is orthogonal to $\phi_{2}, \phi_{3}, \phi_{4}$, which contradicts the fact that the multiplicity of $\lambda_{2}$ is at most three. Therefore we proved (4.39).

Remark 4.5. Since $\phi_{2}$ vanishes at $\mathrm{p}$ with a third order, $\phi_{3}$ vanishes with a second order. From lemma 4.4 and the regularity argument of elliptic equations we know that

$$
\begin{gathered}
\frac{\partial \phi_{2}}{\partial y}(t, p)=c_{4}(t) \frac{\partial \phi_{4}}{\partial y}(p)+O\left(t^{3}\right) . \\
\frac{\partial^{2} \phi_{2}}{\partial x \partial y}(t, p)=c_{3}(t) \frac{\partial^{2} \phi_{3}}{\partial x \partial y}(p)+O\left(t^{2}\right)
\end{gathered}
$$

where $c_{3}(t), c_{4}(t)$ are given in (4.39) and $c_{4}(t)=O\left(t^{2}\right), c_{3}(t)=O(t)$.

In the following, we will rule out case (ii).

Lemma 4.6. Under the assumptions of lemma 4.1, if case (ii) occurs we have

$$
\begin{gathered}
e_{2}(t)=O\left(t^{2}\right) \\
e_{3}(t)=\frac{(\alpha(t)+\beta(t)) t}{\alpha(t)-\beta(t)} e_{3}+O\left(\frac{\alpha(t) t^{2}}{\alpha(t)-\beta(t)}\right)
\end{gathered}
$$

where $e_{3} \neq 0$.

Proof. First we compare $\phi_{4}(t)$ with $\phi_{2}$, as before we have

$$
\begin{aligned}
& \left(\lambda_{2}-\lambda_{4}(t)\right) \int_{\Omega \cap \Omega_{t}} \phi_{4}(t) \phi_{2} \\
& =\frac{1}{2} t^{3}(\alpha(t)-\beta(t)) \frac{\partial \phi_{4}}{\partial y}(t, p) \frac{\partial^{3} \phi_{2}}{\partial x^{2} \partial y}(p) \int_{0}^{1} s^{2} h(s) d s+O\left(\alpha(t) t^{4}\right) .
\end{aligned}
$$


Then from (4.36) and (4.48) we obtain (4.46) easily.

Compare $\phi_{4}(t)$ with $\phi_{3}$, we have

$$
\begin{aligned}
\left(\lambda_{2}-\lambda_{4}(t)\right) \int_{\Omega \cap \Omega_{t}} \phi_{4}(t) \phi_{3}= & t^{2}(\alpha(t)+\beta(t)) \frac{\partial \phi_{4}}{\partial y}(t, p) \frac{\partial^{2} \phi_{3}}{\partial x \partial y}(p) \int_{0}^{1} s h(s) d s \\
& +O\left(\alpha(t) t^{3}\right) .
\end{aligned}
$$

Then from (4.36) and (4.49) we can obtain (4.47) with

$$
e_{3}=\frac{b_{1} \frac{\partial^{2} \phi_{3}}{\partial x \partial y}(p)}{b_{0} \frac{\partial \phi_{4}}{\partial y}(p)} .
$$

Lemma 4.7. Under the assumptions of lemma 4.1, if case (ii) occurs, we have

$$
\left|\begin{array}{ccc}
b_{0} & b_{1} & b_{2} \\
b_{2} & b_{3} & b_{4} \\
b_{1} & 0 & b_{3}
\end{array}\right|=0
$$

where $b_{i}=\int_{0}^{1} s^{i} h(s) d s$.

Proof. From the proof of lemma 4.1, we have

$$
\begin{array}{r}
(\alpha(t)-\beta(t)) \int_{0}^{1}\left(a_{0}(t)+a_{3}(t) t^{2} s^{2}\right) h(s)+(\alpha(t)+\beta(t)) \int_{0}^{1} a_{1}(t) t s h(s)= \\
=O\left(\alpha(t) t^{3}\right)
\end{array}
$$

$$
\begin{array}{r}
(\alpha(t)-\beta(t)) \int_{0}^{1}\left(a_{0}(t) s^{2}+a_{3}(t) t^{2} s^{4}\right) h(s)+(\alpha(t)+\beta(t)) \int_{0}^{1} a_{1}(t) t s^{3} h(s)= \\
=O\left(\alpha(t) t^{3}\right) .
\end{array}
$$

$$
\begin{array}{r}
(\alpha(t)+\beta(t)) \int_{0}^{1}\left(a_{0}(t) s+a_{3}(t) t^{2} s^{3}\right) h(s)+(\alpha(t)-\beta(t)) \int_{0}^{1} a_{1}(t) t s^{2} h(s)= \\
=O\left(\alpha(t) t^{3}\right) .
\end{array}
$$


where $a_{0}(t)=c_{4}(t)+O\left(t^{3}\right), a_{1}(t)=c_{3}(t)+O\left(t^{2}\right)$.

Recall (4.7), where $a_{2}(t)=\frac{\partial^{2} \phi}{\partial y^{2}}(t, p)$, now by lemma 4.4 we can deduce that $a_{2}(t)=O(t)$, then in (4.8) the term $o\left(t^{2}\right)$ can be replaced by $O\left(t^{3}\right)$. Therefore the right hand side of(4.52)-(4.54) become $O\left(\alpha(t) t^{3}\right)$.

Again we assume

$$
\begin{gathered}
\frac{a_{0}(t)}{t^{2}} \longrightarrow x, \\
a_{3}(t) \longrightarrow z, \\
\frac{(\alpha(t)+\beta(t)) a_{1}(t)}{(\alpha(t)-\beta(t)) t} \longrightarrow y,
\end{gathered}
$$

where $y$ is finite. In fact from the domain variation and

$$
\int_{\Omega_{t}} \phi_{2}(t) \phi_{4}(t)=0
$$

we know

$$
\int_{\Omega \cap \Omega_{t}} \phi_{2}(t) \phi_{4}(t)=o\left(t^{5}\right)
$$

Similarly for $i, j=2,3,4$,

$$
\int_{\Omega \cap \Omega_{t}} \phi_{i} \phi_{j}=\delta_{i j}+o\left(t^{5}\right)
$$

Therefore

$$
c_{2}(t) e_{2}(t)+c_{3}(t) e_{3}(t)+c_{4}(t) e_{4}(t)=o\left(t^{5}\right) .
$$

Then from remark 4.5, lemma 3.8 and lemma 4.6, we deduce

$$
\begin{gathered}
c_{3}(t) e_{3}(t)=O\left(t^{2}\right) \\
a_{1}(t)=O\left(\frac{(\alpha(t)-\beta(t)) t}{\alpha(t)}\right) .
\end{gathered}
$$

Then we have

$$
\left\{\begin{array}{l}
b_{0} x+b_{1} y+b_{2} z=0 \\
b_{2} x+b_{3} y+b_{4} z=0 \\
b_{1} x+b_{3} z=0
\end{array}\right.
$$


Since $z \neq 0$, we know the determinant of the coefficient is zero. Then we proved the lemma.

Proof of Theorem 1.1. Suppose the multiplicity of the second eigenvalue is three, then from the above lemmas, we know (4.51) is true. That is

$$
b_{1}\left(b_{2} b_{3}-b_{1} b_{4}\right)-b_{3}\left(b_{0} b_{3}-b_{1} b_{2}\right)=0 .
$$

But by Holder inequality, we already know this is impossible because of $b_{2} b_{3}<b_{1} b_{4}$ and $b_{1} b_{2}<b_{0} b_{3}$. Then we proved the theorem 1.1 .

\section{Appendix.}

We give an elementary proof of an inequality which we mentioned in section 4. We feel indebted to Prof. Liu Jiaqian for providing me the result.

Proposition A. Let $h(s) \geq 0$ in $[0,1]$ and $h(s) \not \equiv 0$, set $b_{i}=\int_{0}^{1} h(s) s^{i} d s$ then we have

$$
b_{2}^{3}+b_{3}^{2} b_{0}+b_{1}^{2} b_{4}<2 b_{1} b_{2} b_{3}+b_{0} b_{2} b_{4} .
$$

Proof. Consider

$$
\int_{0}^{1} \int_{0}^{1} \int_{0}^{1} h(s) h(r) h(t)(s-t)^{2}(s-r)^{2}(t-r)^{2} d s d r d t>0 .
$$

We can obtain the proof of the proposition by expansion of the above inequality.

\section{References.}

[1] N. Aronsajn, A unique continuation theorem for solution of elliptic partial differential equations or inequalities of second order, J. Math. Pure Appl., 36(1957), 235-249.

[2] L. Bers, Local behaviour of solution of general linear elliptic equations, Comm. Pure Appl. Math., 8 (1955), 473-496.

[3] S. Y. Cheng, Eigenfunctions and nodal set, Comm. Math. Helv., 51(1976), 43-55.

[4] R. Courant and D. Hilbert, Methods of mathematical physics, Vol. 1. New York, Interscience 1953. 
[5] C. S. Lin, On the second eigenfunctions of the Laplacian in $R^{2}$, Comm. Math. Phy., 111(1987) 161-166.

[6] C. S. Lin and W. M. Ni, A counterexample to the nodal domain conjecture and a related semilinear equation, Proc. of Amer. Math. Society, Vol 102, No. 2 (1988), 271-277.

[7] C. Miranda, Partial differential equations of elliptic type, Springer-verlag 1970.

[8] A. D. Melas, On the nodal line of the second eigenfunction of the Laplacian in $R^{2}$, J. Diff. Geo., 35(1992), 255-263.

[9] L. E. Payne, Isoperimetric inequalities and their applications, SIAM Rev., 9(1967), 453-488.

[10] S. T. Yau, Problem Section, Seminar On Differential Geometry, Princeton Univ. Press, 1982, 669-706.

RECEIVED OCTOBER 13TH, 1994. 